\title{
Uniform Boundary Stabilization of Quasilinear Wave Equation with Nonlinear Boundary Damping and Source Term
}

\author{
Eugenio Cabanillas Lapa ${ }^{1}$, Victor Emilio Carrera Barrantes ${ }^{2}$, Felix Leon Barboza ${ }^{3}$, Juan Benito
} Bernui Barros ${ }^{4}$ \& Zacarias Huaringa Segura ${ }^{5}$

\begin{abstract}
Resumen: En este trabajo nos refiriremos a la existencia de soluciones fuertes y decaimiento exponencial del total de energía para el problema de condiciones iniciales asociados con la ecuación de onda cuasi-lineal con una fuente no lineal, bajo el supuesto de que la velocidad en la frontera es disipativa. Los resultados son probado por el método de pozo de potencial, la técnica de multiplicadores adecuada y el teorema de la continuación única para la ecuación de onda con coeficientes variables.
\end{abstract}

Palabras clave: Decaimiento exponencial, teorema de la continuación única.

\section{Uniform Boundary Stabilization of Quasilinear Wave Equation with Nonlinear Boundary Damping and Source Term}

\begin{abstract}
In this work we are concerned with the existence of strong solutions and exponential decay of the total energy for the initial boundary value problem associated with the quasilinear wave equation with nonlinear source, under the assumption that the velocity boundary feedback is dissipative. The results are proved by means of the potential well method, the multiplier technique and suitable unique continuation theorem for the wave equation with variable coefficients.
\end{abstract}

Key words: Exponential decay, the unique continuation theorem.

\footnotetext{
${ }^{1}$ UNMSM, Facultad de Ciencias Matemáticas, e-mail: cleugenio@yahoo.com

${ }^{2}$ UNMSM, Facultad de Ciencias Matemáticas, e-mail: vcarrerab@yahoo.com

${ }^{3}$ UNMSM, Facultad de Ciencias Matemáticas, e-mail: fleonb@yahoo.com

${ }^{4}$ UNMSM, Facultad de Ciencias Matemáticas, e-mail: jbernuib@unmsm.edu.pe

${ }^{5}$ UNMSM, Facultad de Ciencias Matemáticas, e-mail: zhuaringas@unmsm.edu.pe
} 


\section{Introducción:}

In this paper we study the global existence and the asymptotic behavior of solutions for the quasilinear wave equation

$$
\begin{gathered}
\left.u_{t t}-\left(a+b \int_{0}^{1} u_{x}^{2} d x\right) u_{x x}=\mu|u|^{q-1} u \quad \text { in }\right] 0,1\left[\times \mathbb{R}^{+}\right. \\
u(0, t)=0, \quad t>0 \\
\left(a+b \int_{0}^{1} u_{x}^{2} d x\right) u_{x}(1, t)=-g\left(u_{t}(1, t)\right), \quad t>0 \\
\left.u(x, 0)=u^{0}(x), \quad u_{t}(x, 0)=u^{1}(x), \quad x \in\right] 0,1[
\end{gathered}
$$

where $a, b$, are positive constants, $q>1, \mu \in \mathbb{R}$ and $g$ is a suitable continuous function.

When $b=0=\mu$ the above equation has been widely studied. For $n \geq 1, a=a(t)$ and MillaMedeiros [6] showed the existence and uniqueness of strong and weak solutions for the problem (1)-(4) .Araruna-Maciel [1] proved the existence an boundary stabilization of the semilinear problem, with a nonlinear function $h$ instead $\mu|u|^{q-1} u$ such that

$$
\operatorname{sh}(s) \leq 0
$$

More recently Cavalcanti et al[3] studied the existence and uniform decay of solutions of (1)(4) subject to a nonlinear feedback acting on the part $\Gamma_{1}$ of the boundary $\Gamma=\Gamma_{0} \cup \Gamma_{1}$. In the quasilinear case (i.e. : $a, b>0$ ) with $\mu=0$ Milla Miranda- Gil Jutuca[7], Lasiecka-Ong [5] ,Cavalcanti et al. [4] ,Ono [8] Tucsnak [9] have studied the problem (1)-(4).

The goal of this work is to state a result of existence and boundary stability of strong solutions to problem (1)-(4)

\section{Notation and statement of results}

We denote

$$
(w, z)=\int_{0}^{1} w(x) z(x) d x, \quad|z|^{q}=\int_{0}^{1}|z(x)|^{q} d x .
$$

For simplicity,we always use $|$.$| to denote |\cdot|_{2}$

By $V$ we denote the Hilbert space

$$
V=\left\{w \in H^{1}(0,1): w(0)=0\right\} .
$$

Now, we set

$$
\begin{aligned}
& J(u)=\frac{a}{2}\left|u_{x}\right|^{2}+\frac{b}{4}\left|u_{x}\right|^{4}-\frac{\mu}{q+1}|u|_{q+1}^{q+1} \\
& I(u)=a\left|u_{x}\right|^{2}-\mu|u|_{q+1}^{q+1}
\end{aligned}
$$

and define the stable set $W$ by

$$
W=\{u \in V: I(u)>0\} \cup\{\theta\}
$$

The energy related to problem (1)-(4) is given by

$$
E(t) \equiv E(u(t))=\frac{1}{2}\left|u_{t}(t)\right|^{2}+J(u(t))
$$


Let $g: \mathbb{R} \rightarrow \mathbb{R}$ be a continuous, monotone, increasing function such that $g(s) . s>0$ for all $s \neq 0$ and

$$
m s^{2} \leq g(s) . s \leq M s^{2} \quad \forall|s| \geq 1, \quad 0<m \leq M
$$

We assume that

$$
\begin{aligned}
& \operatorname{máx}\left\{\frac{2^{5 / 2} b}{a \gamma}\left[\frac{2(q+1)}{a(q-1)} C_{0}\right]^{1 / 2}, \frac{16 C_{*} M^{2}}{a^{3} \gamma} \frac{(q+1) C_{0}}{a(q-1)}\right. \\
& \left.\frac{2 \mu q C_{*}^{q-1}}{\gamma a^{1 / 2}(q-1)}\left[\frac{2(q+1)}{a(q-1)} C_{0}\right]^{(q-1) / 2}+\frac{4 \mu b C_{*}^{q}}{a^{3 / 2} \gamma(q+1)}\left[\frac{2(q+1)}{a(q-1)} C_{0}\right]^{(q+1) / 2}\right\} \\
& <\frac{1}{K}, \text { for } K>3 .
\end{aligned}
$$

where $C_{*}$ is the constant of the imbedding $V \hookrightarrow L^{2 q}(0,1) \hookrightarrow L^{q+1}(0,1)$ and $C_{0}, \gamma$ are positive constants in (16).

We define the function on initial data

$$
F\left(\left|u_{x}^{0}\right|,\left|u_{x x}^{0}\right|,\left|u_{x}^{1}\right|\right)=\frac{1}{2}\left|u_{x}^{1}\right|^{2}+\frac{m_{1}^{2}}{a}\left|u_{x x}^{0}\right|^{2}+\frac{\mu \dot{C}_{*}^{2 q}}{a}\left|u_{x}^{0}\right|_{q+1}^{q+1}
$$

where $m_{1}=a+\frac{2 b(q+1)}{a(q-1)} E(0)$

To get the global existence and regularity for the system (1)-(4) it is natural to deal first with the local existence and uniqueness.In fact, we have the following local result, whose proof is routine and is based on fixed point arguments(See [5] adapted our case)

Teorema 1.1. Suppose that the initial data $u^{0} \in V \cap H^{2}(0,1), u^{1} \in V$ satisfy the compatibility condition

$$
\left(a+b \int_{0}^{1}\left|u_{x}^{0}\right|^{2} d x\right) u_{x}^{0}(1)+g\left(u^{1}(1)\right)=0
$$

Then there exists a number $T(0<T \leq+\infty)$ such that the problem (1)-(4) has a unique solution $u$ on $[0, T[$ with the regularity

$$
u \in C\left(\left[0, T\left[, V \cap H^{2}(0,1)\right) \cap C^{1}\left(\left[0, T[, V) \cap C^{2}\left(\left[0, T\left[, L^{2}(0,1)\right) \diamond\right.\right.\right.\right.\right.\right.
$$

\section{Global Existence and Exponential Decay}

Let $T_{m}$ be the maximal existence time of the solution to the problem (1)-(4).We begin with a basic inequality for a local solution $u(t)$ on $\left[0, T_{m}[\right.$.

Multiplying ((1) by $u_{t}$

$$
\frac{d}{d t} E(t)+g\left(u_{t}(1, t)\right) u_{t}(1, t)=0 .
$$

and integrating from 0 to $t$, we get

$$
E(t)+\int_{0}^{t} g\left(u_{t}(1, t)\right) u_{t}(1, t) d s=E(0)
$$

In particular $E(t)$ is non-increasing on $\left[0, T_{m}[\right.$ and

$$
E(t) \leq E(0)
$$

for all $t \in\left[0, T_{m}[\right.$.

Now ,to obtain a priori estimate, we need the following result 
Lema 1.1. Let $u(t)$ be a solution to the problem (1)-(4) with $u^{0} \in W \cap H^{2}(0,1)$ and $u^{1} \in V$. If

$$
\alpha=\frac{\mu}{a} C_{*}^{q+1}\left[\frac{2(q+1)}{a(q-1)} E(0)\right]^{(q-1) / 2}<1
$$

then $u(t) \in W$,on $\left[0, T_{m}[\right.$.

Prueba. Suppose that there exists a number $\left.t^{*} \in\right] 0, T_{m}\left[\right.$ such that $u(t) \in W$ on $\left[0, t^{*}\left[u\left(t^{*}\right)\right.\right.$ $\notin W$.Then we have

$$
I\left(u\left(t^{*}\right)\right)=0 \text { and } u\left(t^{*}\right) \neq 0
$$

Since $u(t) \in W$ on $\left[0, t^{*}\left[\right.\right.$, it holds $I(u(t)) \geq 0$ on $\left[0, t^{*}\right]$. Then, we deduce that

$$
\begin{aligned}
J(u(t)) & =\frac{a}{2}\left|u_{x}(t)\right|^{2}+\frac{b}{4}\left|u_{x}(t)\right|^{4}-\frac{\mu}{q+1}|u(t)|_{q+1}^{q+1} \\
& =\frac{1}{q-1} I(u(t))+\frac{a(q-1)}{2(q+1)}\left|u_{x}(t)\right|^{2}+\frac{b}{4}\left|u_{x}(t)\right|^{4} \\
& \geq \frac{a(q-1)}{2(q+1)}\left|u_{x}(t)\right|^{2} \quad \text { on }\left[0, t^{*}\right]
\end{aligned}
$$

Consequently,having in mind that $E(t)$ is a non-increasing function,we get

$$
\begin{aligned}
\left|u_{x}(t)\right|^{2} & \leq \frac{2(q+1)}{a(q-1)} J(u(t)) \\
& \leq \frac{2(q+1)}{a(q-1)} E(u(t)) \\
& \leq \frac{2(q+1)}{a(q-1)} E(0) \text { on }\left[0, t^{*}\right]
\end{aligned}
$$

It follows from the Sobolev-Poincaré inequality, the hypothesis and (9) that

$$
\begin{aligned}
\mu|u(t)|_{q+1}^{q+1} & \leq \mu C_{*}^{q+1}\left|u_{x}(t)\right|^{q+1}=\frac{\mu}{a}\left|u_{x}(t)\right|^{q-1} \cdot a\left|u_{x}(t)\right|^{2} \\
& \leq \frac{\mu}{a} C_{*}^{q+1}\left[\frac{2(q+1)}{a(q-1)} E(0)\right]^{(q-1) / 2} \cdot a\left|u_{x}(t)\right|^{2} \\
& <a\left|u_{x}(t)\right|^{2} \text { on }\left[0, t^{*}\right]
\end{aligned}
$$

From (12) and (13) we obtain

$$
\mu|u(t)|_{q+1}^{q+1}<a\left|u_{x}(t)\right|^{2} \text { on }\left[0, t^{*}\right]
$$

Therefore, we obtain

$$
I\left(u\left(t^{*}\right)\right)=a\left|u_{x}\left(t^{*}\right)\right|^{2}-\mu\left|u\left(t^{*}\right)\right|_{q+1}^{q+1}>0
$$

which contradicts to (11). Thus we conclude that $u(t) \in W$,on $\left[0, T_{m}[\right.$.

We shall state our main result 
Teorema 1.2. Suppose that $q>1$ and $\mu>0$. If $u^{0} \in W \cap H^{2}(0,1), u^{1} \in V$ verifying the compatibility condition (7) and

$$
\begin{gathered}
\alpha=\frac{\mu}{a} C_{*}^{q+1}\left[\frac{2(q+1)}{a(q-1)} E(0)\right]^{(q-1) / 2}<1 . \\
F\left(\left|u_{x}^{0}\right|,\left|u_{x x}^{0}\right|,\left|u_{x}^{1}\right|\right)<\frac{\varepsilon_{0}}{K}
\end{gathered}
$$

with $0<\varepsilon_{0}<1$, then the problem (1)-(4) admits a global solution $u=u(x, t)$ satisfying

$$
\mid \begin{aligned}
& u \in L^{\infty}\left(\left[0,+\infty\left[; W \cap H^{2}(0,1)\right)\right.\right. \\
& u_{t} \in L^{\infty}([0,+\infty[; V) \\
& u_{t t} \in L^{\infty}\left(\left[0,+\infty\left[; L^{2}(0,1)\right)\right.\right.
\end{aligned}
$$

Furthermore, the energy determined by the solution $u$ has the following decay rate

$$
E(t) \leq C_{0} e^{-\gamma t}
$$

where $C_{0}$, and $\gamma$ are positive constants .

Prueba. Let $u(t)$ be a unique solution of the problem (1)-(4) in the sense of theorem (1.1) on $\left[0, T_{m}\left[\right.\right.$. We shall show that this solution can be continued to $T_{m}=+\infty$. For this it suffices to derive appropriate apriori estimates including second order derivatives of $u(t)$ and to obtain it we will assume the following lemma to be proven later.

Lema 1.2. For a local solution $u(t)$ of $(1)-(4)$ on $\left[0, T_{m}[\right.$, it holds

$$
E(t) \leq C_{0} e^{-\gamma t}
$$

First of all, we suppose that $\left\{u^{0}, u^{1}\right\}$ are more regular ,e.g. they satisfy

$$
\begin{gathered}
u^{0} \in W \cap H^{3}(0,1), u_{x x}^{0} \in V, u^{1} \in V \cap H^{2}(0,1) \\
\left(a+b \int_{0}^{1}\left|u_{x}^{0}\right|^{2} d x\right) u_{x}^{0}(1)+g\left(u^{1}(1)\right)=0 \\
u_{x x}^{0}(1)=-\left(a+b \int_{0}^{1}\left|u_{x}^{0}\right|^{2} d x\right)^{-1}\left[2 g^{\prime}\left(u^{1}(1)\right)^{-1} b \int_{0}^{1} u_{x}^{0} u_{x}^{1} d x u_{x}^{0}(1)+\mu\left|u^{0}(1)\right|^{q-1} u^{0}(1)\right] \\
-g^{\prime}\left(u^{1}(1)\right)^{-1} u_{x}^{1}(1)
\end{gathered}
$$

If (1) is divided by

$$
\beta(t)=a+b\left|u_{x}(t)\right|^{2}
$$

and the expression resultantly is differentiated with respect to $t$,it yields

$$
\frac{1}{\beta(t)} u_{t t t}(t)-u_{x x t}(t)=\frac{\beta^{\prime}(t)}{\beta^{2}(t)} u_{t t}(t)+\frac{\mu q}{\beta(t)}|u(t)|^{q-1} u_{t}(t)-\frac{\beta^{\prime}(t)}{\beta^{2}(t)}|u(t)|^{q-1} u(t)
$$

Multiplying equation (17) by $u_{t t}$ and integrating, we get

$$
\begin{aligned}
& \frac{d}{d t} H(t)+\frac{g^{\prime}\left(u_{t}(1, t)\right)}{\beta(t)}\left|u_{t t}(1, t)\right|^{2}=\frac{1}{2} \frac{\beta^{\prime}(t)}{\beta^{2}(t)}\left|u_{t t}(t)\right|^{2}+\frac{k \beta^{\prime}(t)}{\beta^{2}(t)} u_{t}(1, t) u_{t t}(1, t) \\
& +\frac{\mu q}{\beta(t)}\left(|u(t)|^{q-1} u_{t}(t), u_{t t}(t)\right)-\frac{\mu \beta^{\prime}(t)}{\beta^{2}(t)}\left(|u(t)|^{q-1} u(t), u_{t t}(t)\right)
\end{aligned}
$$


where

$$
H(t)=\frac{1}{2 \beta(t)}\left|u_{t t}(t)\right|^{2}+\frac{1}{2}\left|u_{x t}(t)\right|^{2}
$$

Making use of the generalized Hölder inequality,observing that $\frac{q-1}{2 q}+\frac{1}{2 q}+\frac{1}{2}=1$, considering the Sobolev imbedding we have

$$
\begin{aligned}
\left|\left(|u(t)|^{q-1} u_{t}(t), u_{t t}(t)\right)\right| & \leq|u(t)|_{2 q}\left|u_{t}(t)\right|_{2 q}\left|u_{t t}(t)\right| \\
& \leq C_{*}^{q-1}\left|u_{x}(t)\right|^{q-1}\left|u_{x t}(t)\right|\left|u_{t t}(t)\right|
\end{aligned}
$$

and

$$
\left|\left(|u(t)|^{q-1} u(t), u_{t t}(t)\right)\right| \leq|u(t)|_{2 q}^{q}\left|u_{t t}(t)\right| \leq C_{*}^{q}\left|u_{x}(t)\right|^{q}\left|u_{t t}(t)\right|
$$

Combining(18) and (19)-(20) we deduce

$$
\begin{aligned}
& \frac{d}{d t} H(t)+\frac{m}{2 \beta(t)}\left|u_{t t}(1, t)\right|^{2} \leq \frac{2^{3 / 2} b}{a}\left[\frac{2(q+1)}{a(q-1)}\right]^{1 / 2} E(t)^{1 / 2} H(t)^{3 / 2}+ \\
& \frac{16 C_{*} M^{2}}{a^{3}} \frac{(q+1)}{a(q-1)} E(t) H(t)^{2}+\left\{\frac{\mu q C_{*}^{q-1}}{a^{1 / 2}}\left[\frac{2(q+1)}{a(q-1)}\right]^{(q-1) / 2} E(t)^{(q-1) / 2}\right. \\
& \left.+\frac{2 \mu b C_{*}^{q}}{a^{3 / 2}}\left[\frac{2(q+1)}{a(q-1)}\right]^{(q+1) / 2} E(t)^{(q+1) / 2}\right\} H(t)
\end{aligned}
$$

On the other hand,by using the original equation ((1) together with the compatibility conditions on the boundary, we get

$$
\left(u_{t t}(0), v\right)=\left(a+b\left|u_{x}^{0}\right|^{2}\right)\left(u_{x x}^{0}, v\right)+\mu\left(\left|u^{0}\right|^{q-1} u^{0}, v\right) ; \forall v \in V
$$

Since $u^{0} \in H^{2}(0,1)$, the Sobolev's imbedding implies

$$
\left|u_{t t}(0)\right| \leq m_{1}\left|u_{x x}^{0}\right|+\mu C_{*}^{q}\left|u_{x}^{0}\right|^{q}
$$

where $m_{1}=a+\frac{2 b(q+1)}{a(q-1)} E(0)$. Thus, we obtain

$$
\frac{\left|u_{t t}(0)\right|^{2}}{2 \beta(0)} \leq \frac{\left|u_{t t}(0)\right|^{2}}{2 a} \leq \frac{m_{1}^{2}}{a}\left|u_{x x}^{0}\right|^{2}+\frac{\mu C_{*}^{2 q}}{a}\left|u_{x}^{0}\right|^{2 q}
$$

and from definition of $H(t)$ it follows that

$$
H(0) \leq \frac{1}{2}\left|u_{x}^{1}\right|^{2}+\frac{1}{a}\left(m_{1}^{2}\left|u_{x x}^{0}\right|^{2}+\mu C_{*}^{2 q}\left|u_{x}^{0}\right|^{2 q}\right) .
$$

Our next goal is to show that $H(t)$ is bounded for all t greater or equal to zero.Actually,we will prove that

$$
H(t)<\epsilon_{0} \quad \text { for all } t \geq 0
$$

where $\epsilon_{0}$ is defined in (15).In fact,suppose that (23)is not true.Then it will exists a $t^{*}>0$ such that

$$
\left\{\begin{array}{l}
H(t)<\epsilon_{0}, \text { for all } 0 \leq t \leq t^{*} \\
H\left(t^{\star}\right)=\epsilon_{0}
\end{array}\right.
$$


If (21) is integrated from 0 to $t^{*}$ we get

$$
\begin{aligned}
& H\left(t^{*}\right) \leq H(0)+\frac{2^{3 / 2} b}{a}\left[\frac{2(q+1)}{a(q-1)} c_{0}\right]^{1 / 2} \epsilon_{0}^{3 / 2} \int_{0}^{t^{*}} e^{-\gamma s / 2} d s \\
& +\frac{16 C_{*} M^{2}}{a^{3}} \frac{(q+1) c_{0}}{a(q-1)} \epsilon_{0}^{2} \int_{0}^{t^{*}} e^{-\gamma s} d s+\left\{\frac{\mu q C_{*}^{q-1}}{a^{1 / 2}}\left[\frac{2(q+1)}{a(q-1)} c_{0}\right]^{(q-1) / 2} \int_{0}^{t^{*}} e^{-\gamma s(q-1) / 2} d s\right. \\
& \left.+\frac{2 \mu b C_{*}^{q}}{a^{3 / 2}}\left[\frac{2(q+1)}{a(q-1)} c_{0}\right]^{(q+1) / 2} \int_{0}^{t^{*}} e^{-\gamma s(q+1) / 2} d s\right\} \epsilon_{0}
\end{aligned}
$$

By using the function $F$ defined in (6) and the estimate (22) in (25) yields

$$
\begin{aligned}
& H\left(t^{*}\right) \leq F\left(\left|u_{x}^{0}\right|,\left|u_{x x}^{0}\right|,\left|u_{x}^{1}\right|\right)+\frac{2^{5 / 2} b}{a \gamma}\left[\frac{2(q+1)}{a(q-1)} c_{0}\right]^{1 / 2} \epsilon_{0}^{3 / 2} \\
& +\frac{16 C_{*} M^{2}}{a^{3} \gamma} \frac{(q+1) c_{0}}{a(q-1)} \epsilon_{0}^{2}+\left\{\frac{2 \mu q C_{*}^{q-1}}{\gamma a^{1 / 2}(q-1)}\left[\frac{2(q+1)}{a(q-1)} c_{0}\right]^{(q-1) / 2}\right. \\
& \left.+\frac{4 \mu b C_{*}^{q}}{a^{3 / 2} \gamma(q+1)}\left[\frac{2(q+1)}{a(q-1)} c_{0}\right]^{(q+1) / 2}\right\} \epsilon_{0}
\end{aligned}
$$

Combining (5) and (15) with (26), we obtain

$$
H\left(t^{*}\right)<\epsilon_{0}
$$

which is a contradiction with $(24)_{2}$, therefore we reach our aim (23).

From definitition of $H(t)$, we conclude

$$
\frac{1}{2 m_{1}}\left|u_{t t}(t)\right|^{2}+\frac{1}{2}\left|u_{x t}(t)\right|^{2} \leq \epsilon_{0} \quad \text { for all } t \geq 0
$$

From (27),system (1)-(4), the classical elliptic theory and trace theory, we get

$$
|u(t)|_{H^{2}} \leq C\left[\left|u_{t t}(t)\right|+\left|u_{x t}(t)\right|\right] \leq C \epsilon_{0}
$$

Then,we conclude that the local solution $u(t)$ with $u(0)=u^{0}, u_{t}(0)=u^{1}$ exists in fact on $[0, \infty[$ and it satisfies all of the above estimates on $[0, \infty[$. The proof of theorem is now finished.

Proof of Lemma (1.2) The method used here is based on the contruction of a suitable Lyapunov functional and a new continuation theorem for the wave equation with variable coefficients. Multiplying equation (1) by $x u_{x}$ we get

$$
\begin{aligned}
& \frac{d}{d t}\left(u_{t}(t), x u_{x}(t)\right)=-\frac{1}{2}\left[\left|u_{t}(t)\right|^{2}+\left(a+b\left|u_{x}(t)\right|^{2}\right)\left|u_{x}(t)\right|^{2}\right]+ \\
& \frac{1}{2}\left[u_{t}^{2}(1, t)+\left(a+b\left|u_{x}(t)\right|^{2}\right) u_{x}^{2}(1, t)\right]+\mu\left(|u(t)|^{q-1} u(t), x u_{x}(t)\right)
\end{aligned}
$$

But

$$
\begin{aligned}
\left|\left(|u(t)|^{q-1} u(t), x u_{x}(t)\right)\right| & \leq|u(t)|_{2 q}^{q}\left|u_{x}(t)\right| \\
& \leq|u(t)|_{2}^{(1-\theta) q} C_{*}^{\theta q}\left|u_{x}(t)\right|^{\theta q+1}, 0<\theta<1
\end{aligned}
$$


where we have used the interpolation inequality and the fact that $|u(t)|_{r} \leq C_{*}\left|u_{x}(t)\right|, \forall r$. From Young inequality, we have for all $\varepsilon>0$, that

$$
\left|\left(|u(t)|^{q-1} u(t), x u_{x}(t)\right)\right| \leq c_{\varepsilon}|u(t)|^{2}+\varepsilon k\left|u_{x}(t)\right|^{2}
$$

where $k=(E(0))^{\frac{q(\theta+1)-2}{2[2-(1-\theta) q]}} C_{*}^{\theta q}$.

Now, using (28)-(30) and the boundary condition, we get

$$
\begin{aligned}
\frac{d}{d t}\left(u_{t}(t), x u_{x}(t)\right) \leq & -\frac{c_{0}}{2}\left[\left|u_{t}(t)\right|^{2}+\left(\frac{a}{2}+\frac{b}{4}\left|u_{x}(t)\right|^{2}\right)\left|u_{x}(t)\right|^{2}\right] \\
& +\frac{1}{2}\left(1+\frac{M^{2}}{a}\right) u_{t}^{2}(1, t)+c_{\varepsilon}|u(t)|^{2}+\varepsilon E(t) \\
\leq & -\frac{c_{0}}{4} E(t)+\frac{1}{2}\left(1+\frac{M^{2}}{a}\right) u_{t}^{2}(1, t)+c_{\varepsilon}|u(t)|^{2}
\end{aligned}
$$

for some $c_{0}>0$.

Our aim now ,is to estimate the last term of (31).In order to obtain it,let us prove the following lemma.

Lema 1.3. There exists $T_{0}>0$ such that if $T \geq T_{0}$,

$$
\int_{S}^{T}|u(t)|^{2} d t \leq C \int_{S}^{T}\left(\left|u_{t}(1, t)\right|^{2}+\left|g\left(u_{t}(1, t)\right)\right|^{2}\right) d t
$$

for $0 \leq S<T<T_{m}$, where $C$ is a positive constant independent of $u$

Prueba. We will argue by contradiction.Let us suppose that (32) is not verified , and so there exists initial data $u^{\nu, 0}$ and $u^{\nu, 1}$ such that the solution $u^{\nu}$ of

$$
\mid \begin{array}{lll}
u_{t t}^{\nu}-\left[a+b \int_{0}^{1}\left(u_{x}^{\nu}\right)^{2} d x\right] u_{x x}=\mu\left|u^{\nu}\right|^{q-1} u^{\nu} & , \quad \text { in }] 0,1[\times] 0,+\infty[ \\
u^{\nu}(0, t)=0 & , \quad \forall t>0 \\
{\left[a+b \int_{0}^{1}\left(u_{x}^{\nu}\right)^{2} d x\right] u_{x}(1, t)=-g\left(u_{t}^{\nu}(1, t)\right)} & , \quad \forall t>0 \\
u^{\nu}(x, 0)=u^{\nu, 0}(x), \quad u_{t}^{\nu}(x, 0)=u^{\nu, 1}(x) & , \quad \forall x \in] 0,1[
\end{array}
$$

where $u^{\nu}$ satisfies

$$
\int_{S}^{T}\left|u^{\nu}(t)\right|^{2} d t>\nu \int_{S}^{T}\left(\left|u_{t}^{\nu}(1, t)\right|^{2}+\left|g\left(u_{t}^{\nu}(1, t)\right)\right|^{2}\right) d t
$$

for any $\nu \in \mathbb{N}$. Here, we observe that in our work, in view of $\alpha<1$, the energy of the initial data $\left\{u^{\nu, 0}, u^{\nu, 1}\right\}$, denoted by $E^{\nu}(0)$, remains uniformly bounded in $\nu$, that is there exists $M>0$ such that $E^{\nu}(0) \leq M, \forall \nu \in \mathbb{N}$. Consequently $E^{\nu}(t) \leq M \quad \forall \nu \in \mathbb{N}$, since it is nonincreasing function. Then we obtain a subsequence, still denoted by $\left\{u^{\nu}\right\}$, which verifies

$$
\begin{aligned}
& u^{\nu} \longrightarrow u \text { weakly } * \text { in } L^{\infty}\left(0, T ; H^{1}(0,1)\right) \\
& u_{t}^{\nu} \longrightarrow u_{t} \text { weakly } * \text { in } L^{\infty}\left(0, T ; L^{2}(0,1)\right) \\
& u_{t}^{\nu}(1, .) \longrightarrow u_{t}(1, .) \text { weak in } L^{2}(0,1)
\end{aligned}
$$

Applying compactness results we deduce that

$$
u^{\nu} \longrightarrow u \text { strongly in } L^{2}\left(0, T ; L^{2}(0,1)\right)
$$

and

$$
u_{t}^{\nu}(1, .) \longrightarrow u_{t}(1, .) \text { strongly in } L^{2}(0, T)
$$


According to (35) we have that

$$
\left.\left|u^{\nu}\right|^{q-1} u^{\nu} \longrightarrow|u|^{q-1} u \quad \text { a.e } \quad \text { in } \quad\right] 0,1[\times] 0, T[
$$

From the above convergence and since the sequence $\left\{\left|u^{\nu}\right|^{q-1} u^{\nu}\right\}$ is bounded in $L^{2}\left(0, T ; L^{2}(0,1)\right)$ we conclude by Lion's lemma that

$$
\left|u^{\nu}\right|^{q-1} u^{\nu} \longrightarrow|u|^{q-1} u \quad \text { weakly in } L^{2}\left(0, T ; L^{2}(0,1)\right) .
$$

The term $\int_{S}^{T}\left|u^{\nu}(t)\right|^{2} d t$ is bounded since $E^{\nu}(t) \leq M, \quad \forall \nu \in \mathbb{N}, \forall t \geq 0$ and $\left|u^{\nu}(t)\right|^{2} \leq C^{\prime} E^{\nu}(t)$ where $C^{\prime}$ is a positive constant independent of $\nu$ and $t$.Then from (34)

$$
\int_{S}^{T}\left(\left|u_{t}^{\nu}(1, t)\right|^{2}+\left|g\left(u_{t}^{\nu}(1, t)\right)\right|^{2}\right) d t \longrightarrow 0 \quad \text { as } \quad \nu \longrightarrow+\infty
$$

As $S$ is chosen in the interval [0,T[,we have

$$
\int_{0}^{T}\left|u_{t}^{\nu}(1, t)\right|^{2} d t \longrightarrow 0 \quad \text { as } \quad \nu \longrightarrow+\infty
$$

and

$$
\int_{0}^{T}\left(\left|g\left(u_{t}^{\nu}(1, t)\right)\right|^{2}\right) d t \longrightarrow 0 \quad \text { as } \quad \nu \longrightarrow+\infty
$$

Besides,from the uniqueness of the limit we conclude that

$$
u_{t}(1, t)=0 \quad \text { and } \quad g\left(u_{t}(1, t)\right)=0
$$

Passing to the limit in (33), when $\nu \longrightarrow+\infty$ we get for $u$

$$
\mid \begin{aligned}
& u_{t t}-\left[a+b \lambda^{2}(t)\right] u_{x x}-\mu|u|^{q-1} u=0 \\
& u(0, t)=0 \\
& u_{x}(1, t)=0, \quad u_{t}(1, t)=0
\end{aligned},
$$

where $\lim _{\nu \rightarrow+\infty} \int_{0}^{1}\left(u_{x}^{\nu}\right)^{2} d x=\lambda^{2}(t)$,by the Ascoli-Arzela Theorem and the boundedness of $E^{\nu}(t)$ (for a subsequence $\left\{u^{\nu_{k}}\right\}$ still denoted by $\left\{u^{\nu}\right\}$ ).

Let $w=u_{t}$. Then

$$
\mid \begin{aligned}
& w_{t t}-\xi(t) w_{x x}=q|u|^{q-1} w+\frac{\xi^{\prime}(t)}{\xi(t)} w_{t}-\frac{\mu \xi^{\prime}(t)}{\xi(t)}|u|^{q-1} u \equiv F(t) \\
& w(0, t)=0=w(1, t) \\
& w_{x}(1, t)=0
\end{aligned},
$$

where $\xi(t)=a+b \lambda^{2}(t)$.

Now,we shall prove a unique continuation property of the problem (40). It is easy to see that the equality (28) can be applicable to the solution $w=u_{t}$ of the problem (40), in place of $u$. Hence , using the boundary conditions, we obtain

$$
\frac{d}{d t}\left(w_{t}(t), x w_{x}(t)\right) \leq-\frac{c_{0}}{4} E_{1}(t)+\left(F(t), x w_{x}\right)
$$

where $E_{1}(t)=\frac{1}{2}\left(\left|u_{t t}(t)\right|^{2}+\xi(t)\left|u_{x t}(t)\right|^{2}\right)$.

Here, we observe that $Q(t)=\left(w_{t}(t), x w_{x}(t)\right.$ verifies

$$
q_{0} E_{1}(t) \leq Q(t) \leq q_{1} E_{1}(t)
$$


where $q_{0}$ and $q_{1}$ are positive constants, $q_{0}<q_{1}$. Thus, using (42) we have

$$
E_{1}(T)+c_{0}^{1} \int_{0}^{T} E_{1}(t) d t \leq c\left(E_{1}(0)+\int_{0}^{T}|F(t)|\left|w_{x}(t)\right| d t\right)
$$

and hence

$$
\int_{0}^{T} E_{1}(t) d t \leq c\left(E_{1}\left(t^{*}\right)+\int_{0}^{T}|F(t)|^{2} d t\right)
$$

where $E_{1}\left(t^{*}\right)=\inf _{0 \leq t \leq T} E_{1}(t)$.

Here, we have

$$
\begin{gathered}
|F(t)|^{2} \leq C\left(\left.\left.|| u\right|^{q-1} w\right|^{2}+\left|\frac{\xi^{\prime}(t)}{\xi(t)}\right|^{2}\left|w_{t}\right|^{2}+\left.\left.\left|\frac{\mu \xi^{\prime}(t)}{\xi(t)}\right|^{2}|| u\right|^{q-1} u\right|^{2}\right) \\
\left.\left.|| u\right|^{q-1} w\right|^{2} \leq|u|_{\infty}^{2(q-1)}|w|^{2} \leq C\left|u_{x}\right|^{2(q-1)}\left|w_{x}\right|^{2} \leq \frac{C E(0)^{q-1}}{a} \frac{\xi(t)}{2}\left|w_{x}\right|^{2}
\end{gathered}
$$

Further, by the equation we see

$$
\xi(t)\left|u_{x}(t)\right|^{2} \leq C_{1}\left(\left|u_{t t}(t)\right|+\left|u_{x t}(t)\right|+\left|u_{x}(t)\right|^{q}\right)\left|u_{x}(t)\right|
$$

with some $C_{1}>0$,we obtain

$$
\left(1-\frac{C_{1}}{a} E(0)^{(q-1) / 2}\right)\left|u_{x}(t)\right| \leq \frac{C_{1}}{a}\left(\left|u_{t t}(t)\right|+\left|u_{x t}(t)\right|\right)
$$

Thus,under a little more stronger assumption than (10)

$$
\alpha+\frac{C_{1}}{a} E(0)^{(q-1) / 2}<1
$$

we get

$$
\left|u_{x}(t)\right| \leq C E_{1}^{1 / 2}(t)
$$

Then

$$
\begin{aligned}
\left.\left.|| u(t)\right|^{q-1} u(t)\right|^{2} \leq|u(t)|_{2 q}^{2 q} \leq C_{*}^{2 q}\left|u_{x}(t)\right|^{2 q} & \leq C E(0)^{q-1}\left|u_{x}(t)\right|^{2} \\
& \leq C E_{1}(t)
\end{aligned}
$$

Furthermore,by the assumptions, we have

$$
\begin{aligned}
\left|\frac{\xi^{\prime}(t)}{\xi(t)}\right| & \leq \frac{1}{a} \lim _{\nu \rightarrow+\infty}\left|\frac{d}{d t}\left[a+b \int_{0}^{1}\left(u_{x}^{\nu}\right)^{2} d x\right]\right|=\frac{2 b}{a} \lim _{\nu \rightarrow+\infty}\left|\int_{0}^{1} u_{x}^{\nu} u_{x t}^{\nu} d x\right| \\
& \leq \frac{2 b}{a}\left|u_{x}(t)\right|\left|u_{x t}(t)\right| \leq \frac{2^{3 / 2} b}{a K^{1 / 2}}\left[\frac{2(q+1)}{a(q-1)} E(0)\right]^{1 / 2} \epsilon_{0}^{1 / 2},
\end{aligned}
$$

on $\left[0, T_{m}[\right.$. Then we have from (43)-(48)

$$
\int_{0}^{T} E_{1}(t) d t \leq c\left(E_{1}\left(t^{*}\right)+\epsilon_{0} \int_{0}^{T} E_{1}(t) d t\right)
$$


taking $\epsilon_{0}$ small we arrived at the inequality

$$
\int_{0}^{T} E_{1}(t) d t \leq C_{2} E_{1}\left(t^{*}\right)
$$

for a certain constant $C_{2}>0$. Taking $T>T_{0} \equiv C_{2}$ we obtain $E_{1}(t)=0 \quad, 0 \leq t \leq T$ , which implies $u(x, t)=u(x)$, independent of $t$. So, the original problem (39) implies

$$
a\left|u_{x}(t)\right|^{2} \leq \mu|u|_{q+1}^{q+1}
$$

But, this contradicts the lemma 1.1 if $u \neq 0$. Here we observe that we may assume $T_{m}>T_{0}$. Otherwise,we get the results by (9). Let us assume that $u=0$. Defining

$$
\lambda_{\nu}^{2}=\int_{S}^{T}\left|u^{\nu}\right|^{2} d s \quad, \quad z^{\nu}(x, t)=\frac{u^{\nu}(x, t)}{\lambda_{\nu}} \quad, \quad 0 \leq t \leq T
$$

we have that $\lambda_{\nu} \rightarrow 0$ and

$$
\int_{S}^{T}\left|z^{\nu}\right|^{2} d s=1
$$

Besides

$$
\begin{aligned}
\widetilde{E}^{\nu}(t) & \equiv E\left(z^{\nu}(t)\right)=\frac{1}{2}\left|z_{t}^{\nu}(t)\right|^{2}+J\left(z_{t}^{\nu}(t)\right. \\
& \leq \frac{1}{2}\left|z_{t}^{\nu}(t)\right|^{2}+\frac{a}{2}\left|z_{x}^{\nu}(t)\right|^{2}+\frac{b}{4}\left|z_{x}^{\nu}(t)\right|^{4} \\
& \leq \frac{1}{2 \lambda_{\nu}^{2}}\left\{\left|u_{t}^{\nu}(t)\right|^{2}+a\left|u_{x}^{\nu}(t)\right|^{2}+\frac{b}{2}\left|u_{x}^{\nu}(t)\right|^{4}\right\}
\end{aligned}
$$

Then

$$
\widetilde{E}^{\nu}(t) \leq \frac{1}{\lambda_{\nu}^{2}}\left(\frac{q+1}{q-1}\right) E^{\nu}(t)
$$

Also

$$
\begin{aligned}
\tilde{E}^{\nu}(t) & \geq \frac{1}{2}\left\{\left|z_{t}^{\nu}(t)\right|^{2}+\frac{a(q-1)}{q+1}\left|z_{x}^{\nu}(t)\right|^{2}+\frac{b}{2}\left|z_{x}^{\nu}(t)\right|^{4}\right\} \\
& \geq \frac{1}{\lambda_{\nu}^{2}}\left(\frac{q-1}{q+1}\right) E^{\nu}(t)
\end{aligned}
$$

On the other hand,applying inequality (31) to the solutions $\left\{u^{\nu}\right\}_{\nu \geq 1}$ we have

$$
\frac{d}{d t} K^{\nu}(t) \equiv \frac{d}{d t}\left(u_{t}^{\nu}, x u_{x}\right) \leq-\delta_{0} E^{\nu}(t)+C_{3}\left(\left|u_{t}^{\nu}(1, t)\right|^{2}+c_{\varepsilon}\left|u^{\nu}(t)\right|^{2}\right)
$$

then integrating over $[S . T]$, we obtain

$$
K^{\nu}(T)+\delta_{0} \int_{S}^{T} E^{\nu}(t) d t \leq K^{\nu}(S)+C_{3} \int_{S}^{T}\left(\left|u_{t}^{\nu}(1, t)\right|^{2}+c_{\varepsilon}\left|u^{\nu}(t)\right|^{2}\right) d t
$$

Since $K^{\nu}$ satisfies

$$
q_{0} E^{\nu}(t) \leq K^{\nu}(t) \leq q_{1} E^{\nu}(t)
$$


for some $q_{0}, q_{1}>0$, and recalling that $E^{\nu}$ is a decreasing function , we get

$$
E^{\nu}(T)+\left(\delta_{0}-\frac{C_{1}^{\prime}}{T}\right) \int_{S}^{T} E^{\nu}(t) d t \leq C_{3}^{\prime} \int_{S}^{T}\left(\left|u_{t}^{\nu}(1, t)\right|^{2}+\left|u^{\nu}(t)\right|^{2}\right) d t
$$

Dividing both sides of (54) by $\lambda_{\nu}^{2}$, applying inequalities (52),(53),(34) and taking T large enough,we conclude that $E^{\nu}(T)$ is bounded.

From (8),integrating over $[t, T] \subseteq[S, T]$

$$
E^{\nu}(t)=E^{\nu}(T)+\int_{t}^{T} g\left(u_{t}^{\nu}(1, s)\right) u_{t}^{\nu}(1, s) d s
$$

Dividing both sides of this inequality by $\lambda_{\nu}^{2}$, we have

$$
\frac{E^{\nu}(t)}{\lambda_{\nu}^{2}} \leq \frac{q+1}{q-1} \widetilde{E}^{\nu}(T)+\frac{M}{\lambda_{\nu}^{2}} \int_{S}^{T}\left|u_{t}^{\nu}(1, s)\right|^{2} d s
$$

From (34) we deduce that

$$
\lim _{\nu \rightarrow \infty} \frac{M}{\lambda_{\nu}^{2}} \int_{S}^{T}\left|u_{t}^{\nu}(1, s)\right|^{2} d s=0
$$

and consequently, there exists $\hat{M}>0$ such that

$$
\frac{E^{\nu}(t)}{\lambda_{\nu}^{2}} \leq \hat{M}
$$

for all $t \in[S, T]$ and $\nu \in \mathbb{N}$.

From (52) it comes that

$$
\widetilde{E}^{\nu}(t) \leq \hat{M}, \quad t \in[S, T], \nu \in \mathbb{N}
$$

then in particular, for a subsequence $\left\{z^{\nu}\right\}$, we obtain

$$
\begin{aligned}
& z^{\nu} \longrightarrow z \text { weakly } * \text { in } L^{\infty}\left(0, T ; H^{1}(0,1)\right) \\
& z_{t}^{\nu} \longrightarrow z_{t} \text { weakly } * \text { in } L^{\infty}\left(0, T ; L^{2}(0,1)\right) \\
& z^{\nu} \longrightarrow z \text { strongly } \quad \text { in } L^{2}\left(0, T ; L^{2}(0,1)\right)
\end{aligned}
$$

In addition $\left\{z^{\nu}\right\}$ satisfies

$$
\mid \begin{aligned}
& z_{t t}^{\nu}-\left[a+b \int_{0}^{1}\left(u_{x}^{\nu}\right)^{2} d x\right] z_{x x}^{\nu}=\mu\left|u^{\nu}\right|^{q-1} z^{\nu} \\
& z^{\nu}(0, t)=0 \\
& {\left[a+b \int_{0}^{1}\left(u_{x}^{\nu}\right)^{2} d x\right] z_{x}(1, t)=-g\left(z_{t}^{\nu}(1, t)\right)}
\end{aligned}
$$

From (55), we obtain (for $S=0$ )

$$
z_{t}^{\nu}(1, .) \longrightarrow 0 \quad \text { in } L^{2}(0, T) \text { as } \nu \longrightarrow+\infty
$$

In addition, using the same idea as in [2] we prove

$$
\mu\left|u^{\nu}\right|^{q-1} z^{\nu} \longrightarrow 0 \quad \text { in } L^{2}\left(0, T ; L^{2}(0,1)\right) \text { as } \nu \longrightarrow+\infty
$$

Passing to the limit in (56) as $\nu \longrightarrow+\infty$, taking (58) and hypothesis on $g$ into account we have

$$
\mid \begin{aligned}
& z_{t t}-\xi(t) z_{x x}^{\nu}=0 \\
& z^{\nu}(0, t)=0 \\
& z_{x}(1, t)=0=z_{t}^{\nu}(1, t)
\end{aligned}
$$


Repeating the above procedure in the case $u \neq 0$, taking $\mu=0$, we get $z=0$ which contradicts (50).

So, lema 1.3 is proved.

Now, we consider the functional

$$
Q(t)=E(t)+\epsilon\left(u_{t}(t), x u_{x}(t)\right)
$$

with $\epsilon>0$.We observe that $Q(t)$ satisfies

$$
\widehat{q}_{0} E(t) \leq Q(t) \leq \widehat{q}_{1} E(t)
$$

Then ,from (8),(31),integrating from $\mathrm{S}$ to $\mathrm{T}, 0 \leq S \leq T<\infty$, using (32),(59) and choosing $\epsilon>0$ sufficiently small,we obtain

$$
\int_{S}^{T} E(t) d t \leq C E(S)
$$

which proves lemma 1.2. 


\section{REFERENCIAS BIBLIOGRÁFICAS}

[1] F.D.Araruna .,A.B. Maciel . Existence an boundary stabilization of the semilinear wave equation,.Nonlinear Analysis (2007)Vol 67,4,1288-1305.

[2] E. Cabanillas Lapa:,Z. Huaringa S., B Godoy T..,The transmission problem with nonlinear damping and source terms,Appl.Math and Inf. Sc.Int. J.,2(2) (2008),187-209

[3] M. Cavalcanti .,V.Domingos CavalcantiV, J.A.Soriano .; Existence and boundary stabilization of a nonlinear hyperbolic equation with time dependent coefficients, Elec J. Diff. Eq.8(1998),121.

[4] M.Cavalcanti ,V.Cavalcanti , J.ASoriano,Global existence and uniform decay rates of the Kirchhoff-Carrier with nonlinear dissipation ,Adv. Diff. Eq.Vol 6,6,2001,701-730.

[5] I.Lasiecka ,J.Ong ; Global solvability and uniform decay of solutions to quasilinear equation with nonlinear boundary dissipation,Comm.Part.Diff. Eq.,24 (1999),2069-2107

[6] M.Milla Miranda , L. A.Medeiros ; On a boundary value problem for wave equation: Existence,uniqueness, asymptotic behavior,Rev. Mat. Ap. Univ.de Chile,17(1996),47-73.

[7] M.Milla Miranda ,L.San Gil Jutuca . ; Existence and boundary stability of solutions for the Kirchhoff equation,Comm. Part. Diff. Eq.,24(1999),1759-1800

[8] K.Ono . ; A stretched string equation with a boundary dissipation,Kyushu J. Math,48(1994),265-281

[9] M.Tucsnak .;Boundary stabilization for the stretched string equation,Diff. Integ. Eq. ,6(1993),925-935 